\title{
Dextromethorphan provides neuroprotection via anti-inflammatory and anti-excitotoxicity effects in the cortex following traumatic brain injury
}

\author{
BENFANG PU*, YONGHUA XUE*, QINGMING WANG, CHUNHUI HUA and XINYUAN LI \\ Department of Neurosurgery, Tongren Hospital Affiliated to Shanghai Jiaotong University School of Medicine, \\ Shanghai 200333, P.R. China
}

Received August 14, 2014; Accepted April 28, 2015

DOI: $10.3892 / \mathrm{mmr} .2015 .3830$

\begin{abstract}
Traumatic brain injury (TBI) is caused by primary and secondary injury mechanisms. TBI induces a certain amount of inflammatory responses and glutamate excitotoxicity that are believed to participate in the pathogenesis of secondary injury. The non-narcotic anti-tussive drug dextromethorphan (DM) has been reported to have a high safety profile in humans and its neuroprotective against a variety of disorders, including cerebral ischemia, epilepsy and acute brain injury. However, few studies have explored the underlying mechanisms of the neuroprotective effects of DM in animals in the setting of TBI. The aim of the present study was to investigate the neuroprotective effects of DM on TBI and to determine the underlying mechanisms. Rats were subjected to a controlled cortical impact (CCI) injury and randomly divided into three groups: Sham-operated, TBI and DM treatment groups. The DM treatment group was administered DM $(30 \mathrm{mg} / \mathrm{kg}$ of body weight, intraperitoneally) immediately after injury. It was identified that DM treatment following TBI significantly reduced brain edema and neurological deficits, as well as increased neuronal survival. These effects correlated with a decrease of tumor necrosis factor $\alpha$, interleukin-1 $\beta$ (IL-1 $\beta$ ) and IL-6 protein expression and an increase of glutamate/aspartate transporter and glutamate
\end{abstract}

Correspondence to: Dr Xinyuan Li, Department of Neurosurgery, Tongren Hospital Affiliated to Shanghai Jiaotong University School of Medicine, 1111 Xianxia Road, Shanghai 200333, P.R. China E-mail: leexinyuan24k@163.com

*Contributed equally

Abbreviations: DM, dextromethorphan; TBI, traumatic brain injury; CCI, controlled cortical impact; NSS, neurologic severity score; NeuN, neuron-specific nuclear protein; TNF- $\alpha$, tumor necrosis factor $\alpha$; IL-6, interleukin-6; IL-1 $\beta$, interleukin-1 $\beta$; GLAST, glutamate/aspartate transporter; GLT-1, glutamate transporter-1

Key words: dextromethorphan, neuroprotection, traumatic brain injury, inflammatory, excitotoxicity, rats transporter-1 in the cortex of the brain. These results provided in vivo evidence that DM exerts neuroprotective effects via reducing inflammation and excitotoxicity induced following TBI. The present study has shed light on the potential use of $\mathrm{DM}$ as a neuroprotective agent in the treatment of cerebral injuries.

\section{Introduction}

Traumatic brain injury (TBI), a major public health problem globally, is the leading cause of mortality and morbidity in adults and children (1). TBI is caused by primary as well as secondary injury mechanisms. Primary damage is as a result of mechanical factors immediately following trauma, while secondary injury is produced by complicating processes which are initiated at the moment of impact but do not present clinically for a period of time. Moreover, TBI induces an amount of inflammatory responses that are believed to participate in the pathogenesis of secondary injury (2). In particular, these inflammatory responses incorporate the upregulation of adhesion cytokines, permeation of neutrophils and macrophages as well as activation of glia and neurons (3). Tumor necrosis factor $\alpha(\mathrm{TNF}-\alpha)$, interleukin-1 $\beta$ (IL-1 $\beta$ ) and IL-6 are crucial pro-inflammatory cytokines involved in the inflammatory responses after TBI (4-6). In the serum and cerebral spinal fluid of TBI patients and in brain parenchyma of animals with experimental brain injuries, elevated levels of these cytokines have been detected (7-9). In spite of the potential pathophysiological function of these cytokines in TBI, their role has remained controversial. Evidence from animal experiments has implied that in the initial post-trauma period, elevated levels of TNF- $\alpha$, IL-1 $\beta$ and IL- 6 are harmful, and suppressing the expression of these cytokines may reduce tissue damage and brain edema, and improve the functional outcome $(3,10)$.

Furthermore, multiple previous studies have shown that glutamate is the major excitatory neurotransmitter in the brain $(2,11)$. Accumulation of additional extracellular glutamate and succeeding overstimulation of glutamatergic receptors increases the production of excitotoxic oxygen/nitrogen species, which induce oxidative stress resulting in neuronal death (12). Moreover, high-affinity 
glutamate transporters are able to clear the majority of the glutamate from the extracellular space under physiological conditions (13). There are five different proteins within the glutamate transporter family in the mammalian central nervous system (13). Excitatory amino acid transporter (EAAT)1 and EAAT2, known as glutamate/aspartate transporter (GLAST) and glutamate transporter-1 (GLT-1), respectively, are predominantly expressed in astrocytes and account for $80 \%$ of the total glutamate uptake in the brain (14). The potential of GLAST and GLT-1 to limit extracellular glutamate levels makes them a potential target in diseases associated with glutamate excitotoxicity $(11,15)$. Therefore, a pharmacological approach aimed at increasing glutamate transporter protein levels may be an effective strategy for TBI treatment.

Dextromethorphan (DM) is a non-narcotic anti-tussive drug that initially attracted attention due to its anti-convulsant and neuroprotective properties (16). Since then, multiple studies have demonstrated that DM has a high safety profile in humans and is neuroprotective in a variety of experimental injury models, including cerebral ischemia, epilepsy, neurodegenerative disorders and acute brain injury (17-19). However, few experiments have explored the underlying mechanism of the neuroprotective effect of DM in animals in the setting of TBI.

To determine the potential mechanism of the neuroprotective effect of DM following TBI, the present study aimed to investigate the hypothesis that DM exerts neuroprotective effects via attenuation of pro-inflammatory cytokines and upregulation of glutamate transporter proteins following TBI in rats.

\section{Materials and methods}

Animals. A total of 150 male Sprague-Dawley rats [obtained from Shanghai Jiaotong University Experimental Animal Center (Shanghai, China)], weighing 280-320 g, were allowed free access to food and water under optimal keeping conditions (12-h light/dark cycle; $22^{\circ} \mathrm{C}$ ) prior to the operation. The study was performed in accordance with the Institutional Guidelines for the Care and Use of Laboratory Animals and was approved by the Shanghai Jiaotong Univeristy School of Medicine (Shanghai, China).

Model of TBI. A previously described controlled cortical impact (CCI) injury procedure was utilized. Rats were anesthetized with sodium pentobarbital (i.p; $50 \mathrm{mg} / \mathrm{kg}$; Solar Biotechology, Beijing, China) and placed in a stereotaxic frame. A 5-mm craniotomy was performed over the left parietal cortex, centred on the coronal suture and $3 \mathrm{~mm}$ lateral to the sagittal suture. Considerable care was taken to avoid injury to the underlying dura. Injury was performed using a pneumatic piston with a rounded metal tip ( $2.5 \mathrm{~mm}$ diameter) that was angled $22.5^{\circ}$ vertically so that the tip was perpendicular to the brain surface at the centre of the craniotomy. A velocity of $4 \mathrm{~m} / \mathrm{s}$ and a deformation depth $2 \mathrm{~mm}$ below the dura were used. The bone flap was immediately replaced and sealed, and the scalp was closed with sutures. The body temperature was monitored throughout the surgery by a rectal probe, and the temperature was maintained at $37.0 \pm 0.5^{\circ} \mathrm{C}$ using a heated pad. Rats were placed in a heated cage to maintain body temperature while recovering from anesthesia. Rats, which revealed no symptoms following TBI were excluded from further experiments.

Group and drug administration. Rats were randomly assigned to a sham-operated group (sham; $n=30$ ), a group which received TBI only and which was treated with equal volumes of $0.9 \%$ saline solution (vehicle; $n=60$ ), and a TBI group treated with DM (DM; n=60). DM was dissolved in $0.9 \%$ saline and stored at $4^{\circ} \mathrm{C}$. After brain injury, DM was immediately administered by intraperitoneal injection in the DM group following TBI (30 mg/kg body weight). All tests were run in a blinded manner, and the animal codes were revealed only at the end of the behavioral and histological analyses.

Evaluation of brain edema. Brain edema were evaluated by analysis of the brain water content as described previously (2). Rat brains were separated and weighed immediately with a chemical balance to determine the wet weight (WW). Following drying in a desiccating oven for $24 \mathrm{~h}$ at $100^{\circ} \mathrm{C}$, dry tissues were weighed again to determine the constant dry weight (DW). The percentage of water in the tissues was calculated according to the formula: \% Brain water $=(\mathrm{WW}-\mathrm{DW}) / \mathrm{WW}) \times 100$.

Recovery of motoric function. The neurobehavioral status of the rats was evaluated using a set of 10 tasks, collectively termed Neurologic Severity Score (NSS), which tests reflexes, alertness, coordination and motoric abilities (20). One point is awarded for failure to perform a particular task; thus, a score of 10 reflects maximal impairment, whereas a normal rat scores 0 . Post-injury, the NSS was evaluated at 1, 3 and 5 days. Each animal was assessed by an observer who was blinded to the animal treatment. The difference between the initial NSS and that at any later time was calculated for each rat, and this value $(\triangle \mathrm{NSS})$ reflected the spontaneous or treatment-induced recovery of motoric function.

Immunofluorescence. Brain tissues were fixed in $4 \%$ paraformaldehyde (Solar Biotechology) for $24 \mathrm{~h}$ and immersed in $30 \%$ sucrose solution (Solar Biotechology) with $0.1 \mathrm{~mol} / 1$ phosphate-buffered saline (PBS; pH 7.4; Solar Biotechnology) until sinking to the bottom. Tissue samples $200 \mu \mathrm{m}$ apart from each section from the anterior to the posterior cortex (bregma -1.90 to $-3.00 \mathrm{~mm}$ ) obtained from the TBI rats and embedded in optimal cutting temperature resin. $15 \mu \mathrm{m}$ frozen sections were sliced with a frozen slicer microtome (Leika CM1950; Leika, Mannheim, Germany), treated with $0.4 \%$ Triton-100 for $10 \mathrm{~min}$ and blocked in normal donkey serum for $1 \mathrm{~h}$. The frozen sections were incubated with mouse anti-neuron-specific nuclear protein (NeuN) polyclonal antibody (Santa Cruz Biotechnology, Dallas, TX, USA; diluted 1:100) overnight at $4^{\circ} \mathrm{C}$. The next day, sections were incubated with an anti-mouse immunoglobulin (Ig)G (Santa Cruz Biotechnology; diluted 1:1,000) for $2 \mathrm{~h}$ at $37^{\circ} \mathrm{C}$ in the dark. Images were captured using a laser scanning confocal microscope (Olympus FV1000; Olympus, Tokyo, Japan). Primary antibodies were replaced with PBS in the negative control group.

Western blot analysis. Briefly, rats were anesthetized and underwent intracardiac perfusion with $0.1 \mathrm{~mol} / 1$ phosphate-buffered saline (PBS; pH 7.4). The cortex region of the 


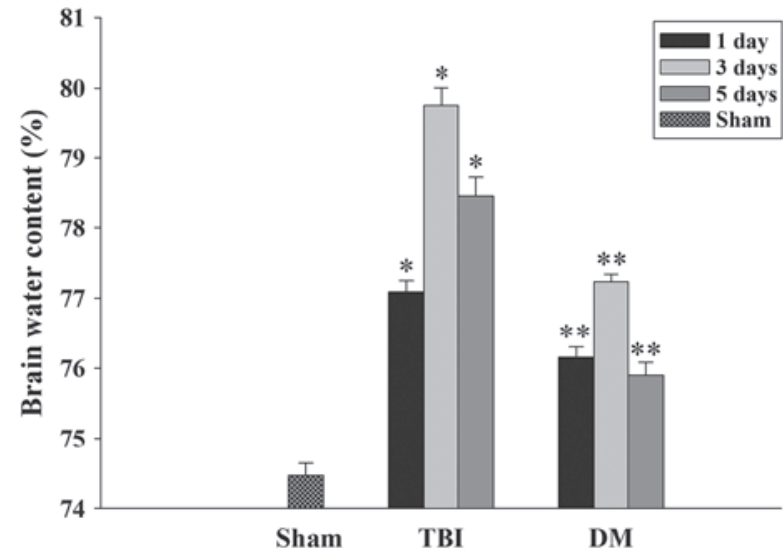

Figure 1. Effect of DM on brain edema. The brain water content was determined at 1, 3 and 5 days following TBI. The brain water content increased markedly at 1,3 and 5 days following TBI ( ${ }^{*} \mathrm{P}<0.01$ vs. sham group). Administration of DM significantly decreased brain edema $\left({ }^{* *} \mathrm{P}<0.05\right.$ vs. TBI group) at 1, 3 and 5 days as reflected by a decrease in brain water content. Values are expressed as the mean \pm standard error ( $n=5$ per group). TBI, traumatic brain injury; DM, dextromethorphan.

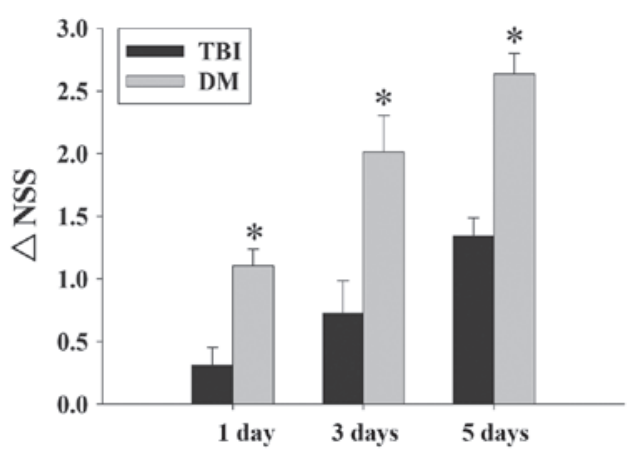

Figure 2. Effect of DM on TBI-induced motor deficits. The temporal changes in motor recovery of the rats were determined at 1,3 and 5 days following TBI and calculated as $\triangle \mathrm{NSS}$. Administration of DM significantly improved motor function at 1,3 and 5 days following TBI ( ${ }^{*} \mathrm{P}<0.01 \mathrm{vs}$. TBI group), as reflected by an increase of $\triangle \mathrm{NSS}$. Values are expressed as the mean \pm standard error ( $n=5$ per group). TBI, traumatic brain injury; DM, dextromethorphan; NSS, neurologic severity score.

brain was rapidly isolated, total protein was extracted and the protein concentration was determined using the bicinchoninic acid method (Solarbio, Beijing, China). Samples were subjected to $30: 0.8 \%$ (w/v) acrylamide/bisacrylamide SDS-PAGE. Separated proteins on the gel were transferred onto polyvinylidene difluoride membranes (Roche Diagnostics, Mannheim, Germany). Blots were blocked with $5 \%$ fat-free dry milk for $1 \mathrm{~h}$ at room temperature. Following blocking, the membrane was incubated with indicated primary antibodies overnight at $4^{\circ} \mathrm{C}$, including rabbit anti-IL-1 $\beta$ polyclonal antibody (Santa Cruz Biotechnology; diluted 1:500), rabbit anti-IL-6 polyclonal antibodies (Santa Cruz Biotechnology; diluted 1:500), rabbit anti-TNF $\alpha$ polyclonal antibody (Santa Cruz Biotechnology; diluted 1:500), rabbit anti-GLAST polyclonal antibody (Santa Cruz Biotechnology; diluted 1:500), rabbit anti-GLT-1 polyclonal antibody (Santa Cruz Biotechnology; diluted 1:500) and mouse anti- $\beta$-actin monoclonal antibody (Santa Cruz Biotechnology; diluted 1:500) overnight at $4^{\circ} \mathrm{C}$. Samples were then incubated with horseradish peroxidase-conjugated anti-rabbit $\mathrm{IgG}$ and anti-mouse IgG (Cell Signaling Technology, Inc., Danvers, MA, USA; diluted 1:5,000) for $2 \mathrm{~h}$ at room temperature. Following incubation with the properly titrated secondary antibody, the immunoblot on the membrane was visible after development with an enhanced chemiluminescence detection system (Aoboxing Biotechnology, Beijing, China) and densitometric signals were quantified using an imaging program. Immunoreactive bands of all proteins expressed were normalized to intensity of corresponding bands for $\beta$-actin. The western blot results were analyzed using ImageJ 1.41 software (National Institutes of Health, Bethesda, MD, USA).

Statistical analysis. All values are expressed as the mean \pm standard deviation. SPSS 16.0 (SPSS, Inc., Chicago, IL, USA) was used for statistical analysis of the data. Statistical analysis was performed using analysis of variance followed by the Student-Newman-Keuls post-hoc tests. $\mathrm{P}<0.05$ was considered to indicate a statistically significant difference between values.

\section{Results}

Treatment with DM attenuates TBI-induced cerebral edema. The wet-dry weight method was used to evaluate brain edema. As shown in Fig. 1, the brain water content was significantly increased in the TBI group compared with that in the sham group at 1, 3 and 5 days after TBI. Of note, the tissue water content in the DM treatment group was significantly reduced at 1, 3 and 5 days compared with that in the TBI group at the same time-point.

Treatment with DM attenuates TBI-induced motoric deficits. Fig. 2 depicts the time-dependent changes in the functional recovery of the rats, expressed as $\triangle \mathrm{NSS}$. The results clearly demonstrated that post-injury administration of DM significantly improved motoric function recovery at 1-5 days following TBI.

Treatment with DM attenuates TNF- $\alpha$ levels in the cortex following TBI. The protein levels of TNF- $\alpha$ in the cortex at 1 , 3 and 5 days were measured by western blot analysis (Fig. 3). TNF- $\alpha$ expression was significantly increased at the various time-points in the TBI group compared with that in the sham group. Of note, administration of DM produced a significant reduction in the TBI-induced upregulation of TNF- $\alpha$ expression.

Treatment with DM attenuates $I L-1 \beta$ levels in the cortex following TBI. The protein levels of IL-1 $\beta$ in the cortex at 1,3 and 5 days were measured by western blot analysis. As shown in Fig. 4, IL-1 $\beta$ expression in the sham rat cortex at each time-point following injury was consistently low, while being significantly increased in the TBI group. Of note, the expression of IL-1 $\beta$ in the DM group was significantly reduced compared with that in the TBI group at the same time-points.

Treatment with DM attenuates $I L-6$ levels in the cortex following TBI. The protein levels of IL-6 in the cortex at 1, 3 and 5 days were determined by western blot analysis. As 

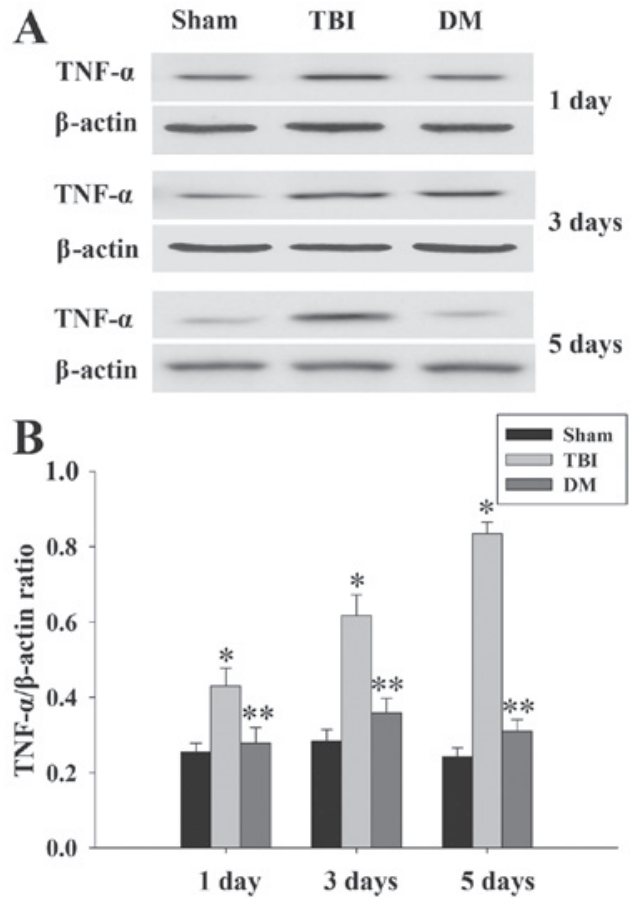

Figure 3. (A) Western blot showing levels of TNF- $\alpha$ in the cortex of rats at 1,3 and 5 days following TBI or sham operation. (B) TNF- $\alpha$ levels were quantified by densitometry against $\beta$-actin bands. The results demonstrated a significant increase of TNF- $\alpha$ expression in the TBI group at 1,3 and 5 days ("P<0.01 vs. sham group). Treatment with DM caused significant downregulation of TNF- $\alpha$ expression at 1,3 and 5 days ( $(* * \mathrm{P}<0.05$ vs. TBI group). Values are expressed as the mean \pm standard error ( $n=5$ per group). TBI, traumatic brain injury; DM, dextromethorphan; TNF- $\alpha$, tumor necrosis factor.

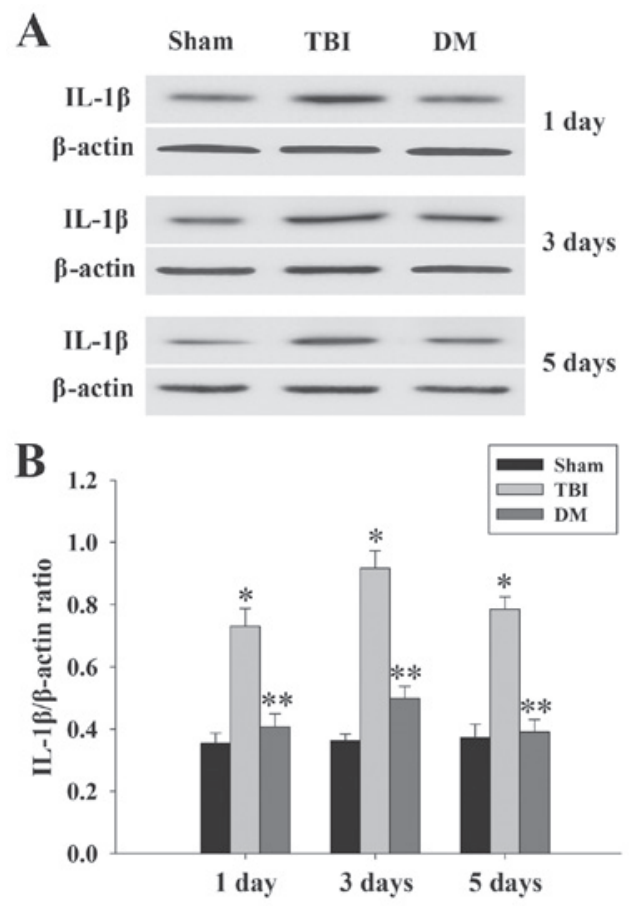

Figure 4. (A) Western blot showing levels of IL-1 $\beta$ in the cortex of rats at 1 , 3 and 5 days following TBI or sham operation. (B) IL- $1 \beta$ levels were quantified by densitometry against $\beta$-actin bands. The results showed a significant increase of IL- $1 \beta$ expression in the TBI group at 1,3 and 5 days (" $\mathrm{P}<0.01$ vs. sham group). DM caused a significant downregulation of IL- $1 \beta$ expression at 1,3 and 5 days $\left({ }^{* *} \mathrm{P}<0.01\right.$ vs. TBI group). Values are expressed as the mean \pm standard error ( $n=5$ per group). TBI, traumatic brain injury; DM, dextromethorphan; IL-1 $\beta$, interleukin-1 $\beta$.
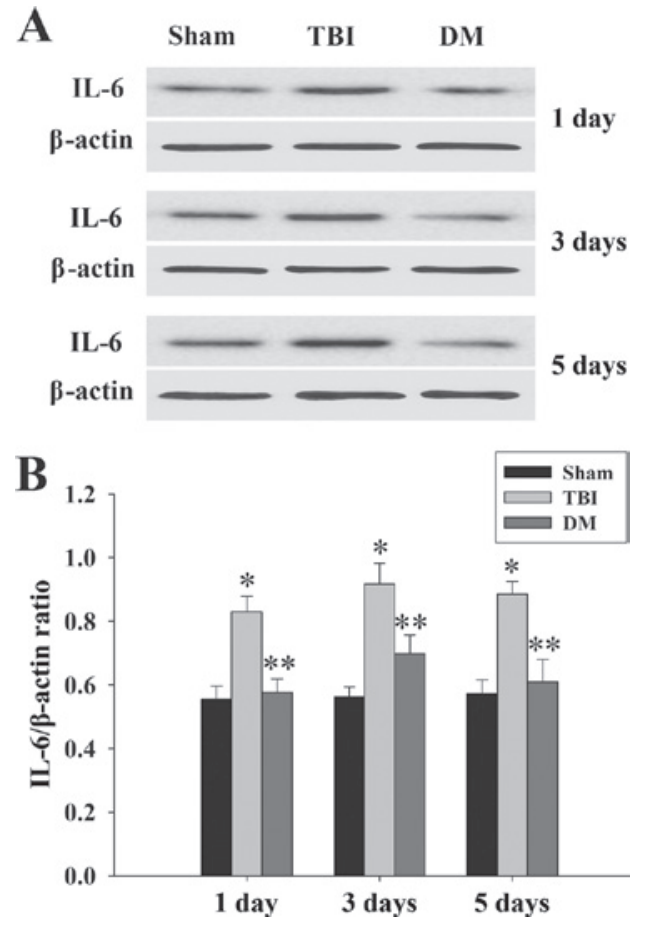

Figure 5. (A) Western blot showing levels of IL-6 in the cortex of rats at 1, 3 and 5 days following TBI or sham operation. (B) Quantitative results of IL-6 expression were determined via the densitometric ratios of IL-6 to $\beta$-actin bands. The results demonstrated a significantly increase of IL-6 expression in the TBI group at 1,3 and 5 days ( $\mathrm{P}<0.05$ vs. sham group). DM caused a significant downregulation of IL- 6 expression at 1,3 and 5 days $\left({ }^{* * *} \mathrm{P}<0.05\right.$ vs. TBI group). Values are expressed as the mean \pm standard error ( $n=5$, per group). TBI, traumatic brain injury; DM, dextromethorphan; IL-6, interleukin-6.
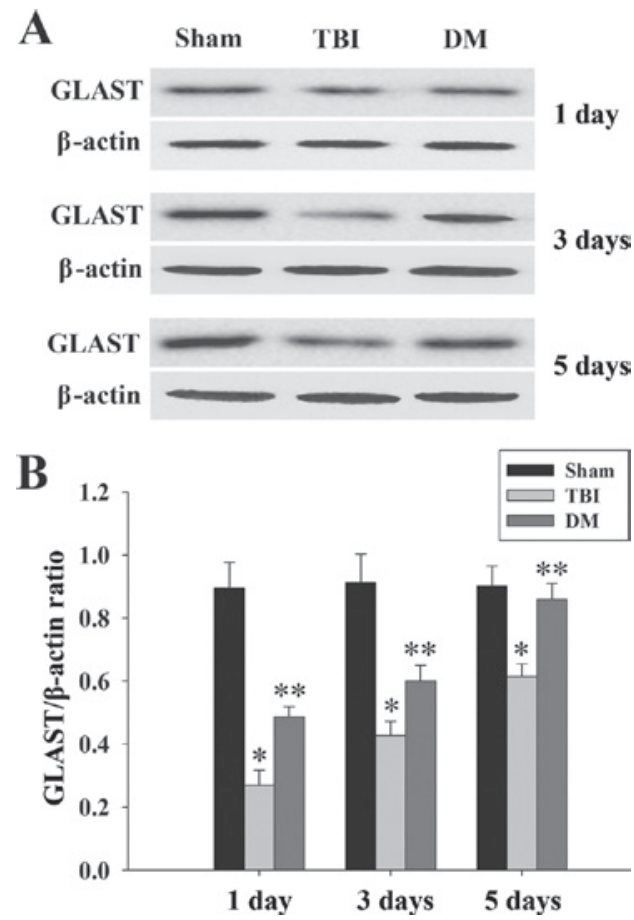

Figure 6. (A) Western blot showing levels of GLAST in the cortex of rats at 1,3 and 5 days following TBI or sham operation. (B) GLAST levels were quantified by densitometry against $\beta$-actin bands. The results demonstrated a significant downregulation of GLAST expression in the TBI group at 1,3 and 5 days (" $\mathrm{P}<0.01$ vs. sham group). DM caused a significant increase of GLAST expression at 1,3 and 5 days $\left({ }^{* *} \mathrm{P}<0.05\right.$ vs. TBI group). Values are expressed as the mean \pm standard error ( $n=5$, per group) TBI, traumatic brain injury; DM, dextromethorphan; GLAST, glutamate/aspartate transporter. 

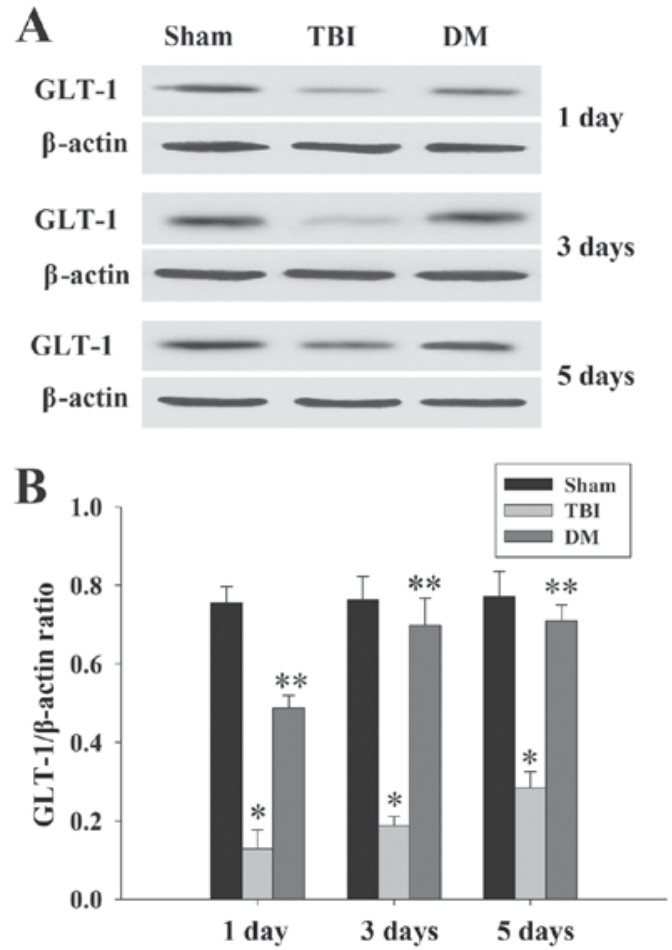

Figure 7. (A) Western blot showing levels of GLT-1 in the cortex of rats at 1,3 and 5 days following TBI or sham operation. (B) GLT-1 levels were quantified by densitometry against $\beta$-actin bands. The results demonstrated a significant downregulation of GLT- 1 expression in the TBI group at 1,3 and 5 days (" $\mathrm{P}<0.01$ vs. sham group). DM caused a significant increase of GLT-1 expression at 1,3 and 5 days ("* $\mathrm{P}<0.05$ vs. TBI group). Values are expressed as the mean \pm standard error ( $n=5$, per group). TBI, traumatic brain injury; DM, dextromethorphan; GLT-1, glutamate transporter-1.

shown in Fig. 5, IL-6 expression was significantly increased at various time-points in the TBI group compared with that in the sham group. By contrast, treatment with DM produced a significant reduction of IL- 6 expression compared with that in the TBI group.

Treatment with DM increases GLAST protein expression in the cortex following TBI. GLAST protein expression in the cortex was determined by western blot analysis at 1,3 and 5 days. As demonstrated in Fig. 6, there was a significant downregulation of GLAST expression in the TBI group compared with that in the sham group. Of note, administration of DM caused a marked elevation of GLAST at 1,3 and 5 days compared to that in the TBI group.

DM treatment increases GLT-1 expression in the cortex following TBI. GLT-1 protein expression in the cortex was assessed by western blot analysis at 1, 3 and 5 days. As demonstrated in Fig. 7, there was a significant downregulation of GLT-1 expression in the TBI group compared to that in the sham group at 1,3 and 5 days. DM produced a marked elevation of GLT-1 expression compared with that in the TBI group at the same time-points.

Treatment with DM increases neuronal survival in the cortex following TBI. The cortex regions of brains were collected at 1 , 3 and 5 days after TBI and subjected to immunostaining with
A
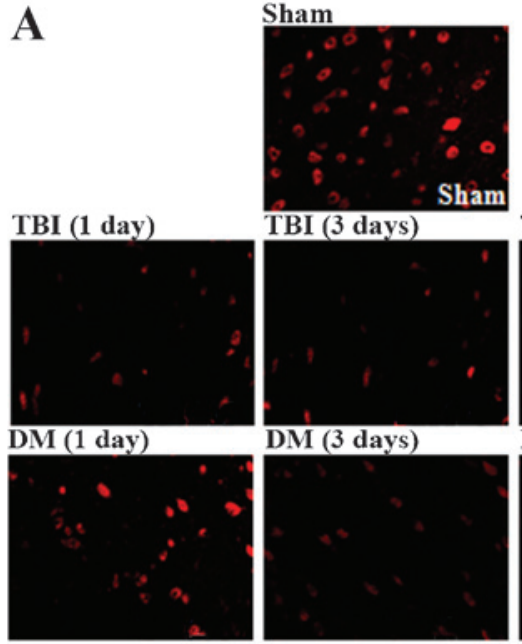

DM (3 days)
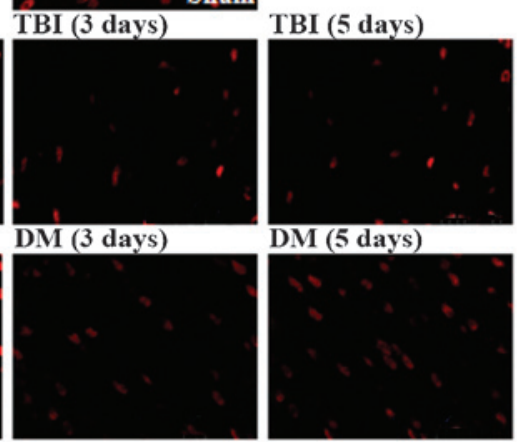

DM (5 days)

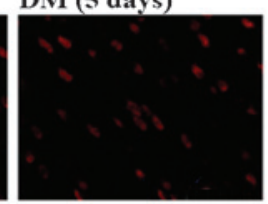

B

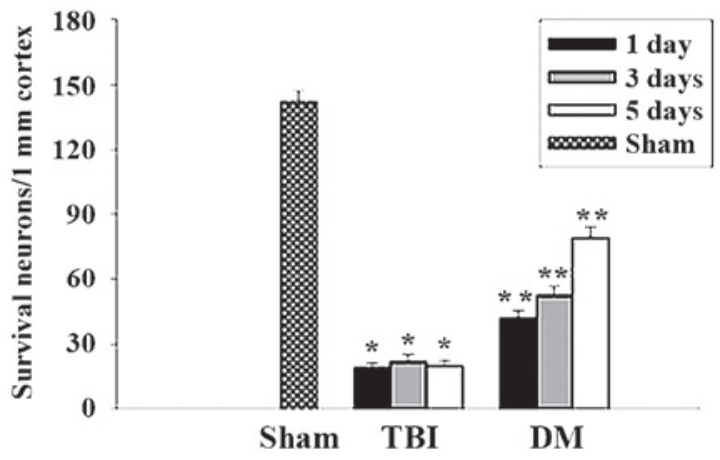

Figure 8. (A) Neuronal survival was quantified using NeuN immunostaining of representative cortex sections 1,3 and 5 days following TBI. (B) Quantitative analysis of the images shows the number of surviving neurons per $1 \mathrm{~mm}$ length of cortex sectors. The results demonstrated that TBI caused a significant increase in the number of surviving neurons at 1,3 and 5 days following TBI (" $\mathrm{P}<0.01$ vs. TBI group), while administration of DM significantly increased neuronal survival in the cortex compared to that in the TBI group at 1, 3 and 5 days. Values are expressed as the mean \pm standard error ( $n=5$, per group) TBI, traumatic brain injury; DM, dextromethorphan; NeuN, neuron-specific nuclear protein.

the neuronal marker NeuN. Neuronal survival was quantified by counting the number of NeuN-positive cells per $1 \mathrm{~mm}$ length in the cortexes of all rats. Representative images of NeuN-stained sections are presented in Fig. 8A, and quantified results from all rats are presented in Fig. 8B. As demonstrated in Fig. 8, at 1, 3 and 5 days following TBI, administration of DM significantly increased neuronal survival in the cortex compared to that in the TBI group.

\section{Discussion}

TBI is the main cause of death in children and young adults worldwide (1). Studies using animal models of TBI are important in the process of understanding and evaluating the complex physiological, behavioral and histopathological changes associated with TBI (21). However, head injury is an unpredictable and spontaneous event, and no single animal model is entirely successful in reproducing the complete pathological changes observed following TBI in humans. In the present study, a CCI model of TBI was used. This model is an invasive impact method that was adapted from 
similar methods employed in experimental spinal cord injury studies. A number of advantages of this model include the ability to control deformation parameters, including velocity, time and depth of impact. In addition, this model can also mimic the whole spectrum of focal-type damage and diffuse axonal injury. Therefore, any mice not showing moderate to severe neurological deficits consistent with the surgery were excluded from further study.

In the present study, the effectiveness of the common anti-tussive agent DM as a therapeutic option for the treatment of TBI was tested. The results showed that a single injection of DM immediately following TBI significantly reduced brain edema and neurological deficits as well as increased neuronal survival. These effects correlated with a decrease of TNF- $\alpha$, IL- $1 \beta$ and IL- 6 protein expression and an increase of GLAST and GLT-1 in the cortex of the brain. Previous studies have demonstrated that DM provides neuroprotection in a variety of experimental injury models, including epilepsy, cerebral ischemia, neurodegenerative disorders and acute brain injury (17-19). Using the rat model of TBI, the present study confirmed and extended these previous observations and demonstrated, for the first time, that post-injury administration of DM provides a neuroprotective function via attenuation of pro-inflammatory cytokines and upregulation of glutamate transporter proteins following experimental TBI in rats.

Inflammatory response induced following TBI is a major contributing factor to secondary injury and has been shown to be an important therapeutic target for reducing the extent of tissue damage after injury (3). TNF- $\alpha$ and IL-1 $\beta$ are potent enhancers of inflammatory reactions via activating blood elements, capillary endothelial cells and glia, as well as through enhancing the expression of downstream inflammatory factors (22). Moreover, these two cytokines also trigger the upregulation of IL-6, which has been suggested to act in concert with TNF- $\alpha$ and IL-1 $\beta$ to mediate multiple biological effects (23). The present study showed, for the first time, that DM suppressed the induction of pro-inflammatory cytokines in the injured brain following TBI. This finding was in agreement with previous studies, which indicated that DM inhibits several inflammatory processes using other animal models $(24,25)$. Although the present study did not establish a direct causal association between cytokine reduction and functional deficits, several lines of evidence implied that TNF- $\alpha$, IL-1 $\beta$ and IL-6 are detrimental in the acute post-injury period and that inhibiting cytokine activation or blocking cytokine receptors may have a neuroprotective effect $(3,10)$.

Excitotoxicity is widely recognized as a crucial process in nerve cell death after acute brain injury (2). Previous studies demonstrated that the neuroprotective properties of DM appear to be functionally associated with its inhibitory effects on glutamate-induced neurotoxicity via its $N$-methyl-D-aspartate receptor antagonist (26), sigma-1 receptor agonist functions (27) and voltage-gated calcium channel antagonist (28). Furthermore, it is worth mentioning that a recent study demonstrated that GLAST and GLT-1 appeared to be inhibited by $\mathrm{MeHg}$ exposure, and these alterations were significantly prevented by pre-treatment with DM (29). However, no previous study has assessed GLAST and GLT-1 expression after DM treatment in a TBI model.
The present study found that GLAST and GLT-1 downregulation was induced after TBI and that this phenomenon was attenuated by administration of DM. These findings emphasized that DM exerts its neuroprotective effects, at least in part via its anti-excitotoxicity effects following experimental TBI in rats.

In conclusion, the present study demonstrated that administration of DM reduced brain edema and neurological deficits as well as increased neuronal survival in a rat model of TBI. Furthermore, DM decreased TNF- $\alpha$, IL- $1 \beta$ and IL-6 protein expression and upregulated GLAST and GLT-1 protein expression in the cortex of the brain. These findings emphasized that DM exerts its neuroprotective function via anti-inflammatory and anti-excitotoxicity effects following experimental TBI in rats. The present study has shed light on the potential use of DM as a neuroprotective agent in the treatment of cerebral injuries.

\section{References}

1. Luo CL, Li BX, Chen XP, et al: Autophagy is involved in traumatic brain injury-induced cell death and contributes to functional outcome deficits in mice. Neuroscience 184: 54-63, 2011.

2. Cui C, Cui Y, Gao J, et al: Neuroprotective effect of ceftriaxone in a rat model of traumatic brain injury. Neurol Sci 35: 695-700, 2014.

3. Morganti-Kossmann MC, Rancan M, Stahel PF and Kossmann T: Inflammatory response in acute traumatic brain injury: a double-edged sword. Curr Opin Crit Care 8: 101-105, 2002 .

4. Feuerstein GZ, Liu T and Barone FC: Cytokines, inflammation and brain injury: role of tumor necrosis factor-alpha. Cerebrovasc Brain Metab Rev 6: 341-360, 1994.

5. Aibiki M, Maekawa S, Ogura S, Kinoshita Y, Kawai N and Yokono S: Effect of moderate hypothermia on systemic and internal jugular plasma IL-6 levels after traumatic brain injury in humans. J Neurotrauma 16: 225-232, 1999.

6. Taupin V, Toulmond S, Serrano A, Benavides J and Zavala F: Increase in IL-6, IL-1 and TNF levels in rat brain following traumatic lesion: Influence of pre-and post-traumatic treatment with Ro5 4864, a peripheral-type (p site) benzodiazepine ligand. J Neuroimmunol 42: 177-185, 1993.

7. Goodman JC, Robertson CS, Grossman RG and Narayan RK: Elevation of tumor necrosis factor in head injury. J Neuroimmunol 30: 213-217, 1990.

8. Csuka E, Morganti-Kossmann MC, Lenzlinger PM, Joller H, Trentz $\mathrm{O}$ and Kossmann T: IL-10 levels in cerebrospinal fluid and serum of patients with severe traumatic brain injury: relationship to IL- 6 , TNF- $\alpha$, TGF- $\beta 1$ and blood-brain barrier function. J Neuroimmunol 101: 211-221, 1999.

9. Hayakata T, Shiozaki T, Tasaki O, et al: Changes in CSF S100B and cytokine concentrations in early-phase severe traumatic brain injury. Shock 22: 102-107, 2004.

10. Lu KT, Wang YW, Yang JT, Yang YL and Chen HI: Effect of interleukin-1 on traumatic brain injury-induced damage to hippocampal neurons. J Neurotrauma 22: 885-895, 2005.

11. Globus MY, Alonso O, Dietrich WD, Busto R and Ginsberg MD: Glutamate release and free radical production following brain injury: effects of posttraumatic hypothermia. J Neurochem 65: 1704-1711, 1995 .

12. Yi JH and Hazell AS: Excitotoxic mechanisms and the role of astrocytic glutamate transporters in traumatic brain injury. Neurochem Int 48: 394-403, 2006.

13. Kanai Y and Hediger MA: Primary structure and functional characterization of a high-affinity glutamate transporter. Nature 360: 467-471, 1992.

14. Pawlak J, Brito V, Küppers E and Beyer C: Regulation of glutamate transporter GLAST and GLT-1 expression in astrocytes by estrogen. Brain Res Mol Brain Res 138: 1-7, 2005.

15. Tanaka K, Watase K, Manabe T, et al: Epilepsy and exacerbation of brain injury in mice lacking the glutamate transporter GLT-1. Science 276: 1699-1702, 1997. 
16. Tortella FC, Britton P, Williams A, Lu XC and Newman AH: Neuroprotection (focal ischemia) and neurotoxicity (electroencephalographic) studies in rats with AHN649, a 3-amino analog of dextromethorphan and low-affinity N-methyl-D-aspartate antagonist. J Pharmacol Exp Ther 291: 399-408, 1999.

17. Werling LL, Lauterbach EC and Calef U: Dextromethorphan as a potential neuroprotective agent with unique mechanisms of action. Neurologist 13: 272-293, 2007.

18. Zhang W, Wang T, Qin L, et al: Neuroprotective effect of dextromethorphan in the MPTP Parkinson's disease model: role of NADPH oxidase. FASEB J 18: 589-591, 2004.

19. Duhaime AC, Gennarelli LM and Boardman C: Neuroprotection by dextromethorphan in acute experimental subdural hematoma in the rat. J Neurotrauma 13: 79-84, 1996.

20. Beni-Adani L, Gozes I, Cohen Y, et al: A peptide derived from activity- dependent neuroprotective protein (ADNP) ameliorates injury response in closed head injury in mice. J Pharmacol Exp Ther 296: 57-63, 2001.

21. Povlishock JT, Hayes RL, Michel ME and Mcintosh TK: Workshop on animal models of traumatic brain injury. J Neurotrauma 11: 723-732, 1994

22. Wang CX and Shuaib A: Involvement of inflammatory cytokines in central nervous system injury. Prog Neurobiol 67: 161-172, 2002.
23. Allan SM and Rothwell NJ: Cytokines and acute neurodegeneration. Nat Rev Neurosci 2: 734-744, 2001.

24. Liu Y, Qin L, Li G, et al: Dextromethorphan protects dopaminergic neurons against inflammation-mediated degeneration through inhibition of microglial activation. J Pharmacol Exp Ther 305: 212-218, 2003.

25. Gao HM, Liu B, Zhang W and Hong JS: Novel anti-inflammatory therapy for Parkinson's disease. Trends Pharmacol Sci 24: 395-401, 2003.

26. Tortella FC, Pellicano M and Bowery NG: Dextromethorphan and neuromodulation: old drug coughs up new activities. Trends Pharmacol Sci 10: 501-507, 1989.

27. Klette KL, Lin Y, Clapp LE, DeCoster MA, Moreton J and Tortella FC: Neuroprotective sigma ligands attenuate NMDA and trans-ACPD-induced calcium signaling in rat primary neurons. Brain Res 756: 231-240, 1997.

28. Carpenter CL, Marks SS, Watson DL and Greenberg DA: Dextromethorphan and dextrorphan as calcium channel antagonists. Brain Res 439: 372-375, 1988.

29. Feng S, Xu Z, Liu W, Li Y, Deng Y and Xu B: Preventive effects of dextromethorphan on methylmercury-induced glutamate dyshomeostasis and oxidative damage in rat cerebral cortex. Biol Trace Elem Res 159: 332-345, 2014. 